\title{
A survey on communication technologies and requirements for internet of electric vehicles
}

\author{
Islam Safak Bayram ${ }^{1 *}$ and loannis Papapanagiotou ${ }^{2}$
}

\begin{abstract}
Electric vehicles (EVs) are becoming a more attractive transportation option, as they offer great cost savings, decrease foreign oil dependency, and reduce carbon emissions. However, varying temporal and spatial demand patterns of EVs threatens power grid operations and its physical components. Thus, the ability of the power grid to handle the potential extra load has become a major factor in the mainstream success. In order for this integration to occur seamlessly, the power grid and the consumers need to be coordinated in harmony. In this paper, we address the critical challenges introduced by the penetration of EVs, systematically categorize the proposed frameworks for demand management, and the role of information and communication technologies in the solution process. We provide a comprehensive survey on the communication requirements, the standards and the candidate technologies towards the Internet of electric vehicles (IoEV). This survey summarizes the current state of research efforts in electric vehicle demand management and aims to shed light on the continued studies.
\end{abstract}

Keywords: Internet of electric vehicles; Smart grid; Demand management; Wireless communications; Networking

\section{Review}

\subsection{Introduction}

As the dependence on a single energy source (crude oil) exposes economies to unstable global oil market and increases environmental concerns, there has been a growing interest to push electric vehicles into mainstream acceptance. The motivation for the electrification of transportation is multifaceted; electricity can be generated through diverse and domestic resources, electricity prices have been relatively stable in the last two decades, and electric miles are cheaper and cleaner [1,2]. Therefore, internet of electric vehicles are expected to achieve a sizable market portion in the next decade. In fact, the study in [3] estimates that there will be around 50 million grid-enabled vehicles by year 2040 .

Accordingly, there is a pressing need in the deployment of charging networks to accommodate the projected demand. For instance, [4] presents that there is an attempt to build a statewide charging station network in California. Similarly, Estonia is building the Europe's largest fast-charging station network with 200 nodes [5].

\footnotetext{
*Correspondence: ibayram@gf.org.qa

${ }^{1}$ Qatar Environment and Energy Research Institute, Qatar Foundation, PO Box 5825, Doha, Qatar

Full list of author information is available at the end of the article
}

The number of EV charging stations is expected exceed four million in Europe and 11 million in the Globe by year 2020 [6].

However, as the power grid is becoming more congested due to the introduction of EVs, managing and controlling of corresponding demand should be carefully aligned with the available resources. Even though, the long term solution involves the upgrade of the power grid components, by considering the potential cost of such investments, the practical solution for the near term would be to develop intelligent control and scheduling techniques to aid the power grid operations. The realization of such frameworks requires appropriate communication architectures that will enable reliable interaction between the grid and the EV drivers to optimally control power flow under varying network conditions.

A handful of surveys have attempted to discuss general smart grid communication requirements, standards, and protocols for household demand management [7-10]. However, the case for the EVs is unique; electric vehicles can be mobile and a typical EV demand is large and, in fact, it can be more than the daily energy consumption of two households [11]. More importantly, the sustainability of the power grid operations is essential for human life. Therefore, careful attention is required to

\section{黑 Springer}


shed more light on the complex problems associated with electrictrification of transportation. Nonetheless, to the best of the authors' knowledge, this is the first study that focuses on the electric vehicle network communications for smart grid applications and, more specifically, to the IoEV challenges. Hence, in this work we

- comprehensively address the unique challenges introduced by the EV penetration specifically for each power grid components and identify opportunities to improve the grid operations and system reliability;

- systematically classify the mathematical frameworks for optimal control and management of EV demand; and

- survey the communication requirements, standards, and candidate technologies that could serve the IoEVs and smart grid applications.

The structure of this paper is as follows. In Section 2, we present the current status of the U.S. power grid, the projected EV roll-out, potential negative impacts on power generation, transmission network, and distribution grid. Next, in Section 3, we categorize the literature on control and coordination frameworks according to the objective function, employed model, and the scale of the problem. In Section 4, we classify published standards and communication technologies respect to each smart grid application. In Section 5, we discuss the communication requirements and performance metrics for the IoEV network communications.

\section{Internet of electric vehicles and the current power grid}

\subsection{Internet of electric vehicles}

Over the last few years, the automotive industry has introduced a variety of new electric vehicle models that have drastically expanded the customer choices [11]. The main drivers that shape the EV adoption include the size of the battery packs (usually varies between 16 to $56 \mathrm{kWh}$ ) and the duration to recharge the vehicle. The battery pack determines the all-electric range of the vehicle and, hence, it is an important criterion to beat the range anxiety. On the other hand, the charging duration depends on the employed charger technology, and it becomes a critical element in order to be competitive against the gas-powered counterparts. For instance, during a charging period of $30 \mathrm{~min}$, level II single, and three-phase, and DC fast charge can enable a Nissan Leaf model (Nissan Motor Co., Ltd., Yokohama, Japan) to drive 5.5, 11 , and 83.4 miles, respectively [12]. The charging standards may vary from country to country, and we present an overview of the different charger standards in Table 1. Moreover, the popularity of each charging type will greatly be determined by the housing demographics [13]. For
Table 1 Electric vehicle charger technologies [14]

\begin{tabular}{lccc}
\hline Type & Connection & Power (kW) & Max current \\
\hline Europe & 1-Phase AC & 3.7 & 16 to 20 \\
Europe & 1 or 3 Phase AC & 3.7 to 22 & 16 to 32 \\
Europe & 3-Phase AC & $>22$ & $>32$ \\
Europe & DC Fast & $>22$ & $>3.225$ \\
USA & AC Level-1 & 1.44 & 12 \\
USA & AC Level-2 & 7.7 & 32 \\
USA & DC Fast & 240 & 400 \\
\hline
\end{tabular}

instance, in the early EV adopter cities, a substantial portion of the population lives in multi-unit dwellings and EVs in these locations will likely use public fast charging facilities. Furthermore, several studies are conducted by different organizations to forecast the $\mathrm{EV}$ penetration rates. Depending on the assumptions made, prediction results may diverge, but nevertheless there is a consensus that EVs will represent a sizable portion in the next decades. In Table 2, the projected EV roll-out is presented. In the rest of this section, we present the current status of the power grid, potential impacts of EV demand, and opportunities offered optimal management of EVs.

\subsection{Power generation and electricity prices}

\subsubsection{Current status}

According to the US National Academy of Engineering, the power grid is 'the supreme engineering achievement of the twentieth century'. Currently, there are close to 3,200 utility companies serving more than 143 million customers in the United States. In order to serve the increasing customer demand, the required power supply is generated through diverse resources, including coal, nuclear, hydro, natural gas, and lately renewable sources, such as wind and solar [16]. Depending on the efficiency and the unit generation cost, power generation can be roughly divided into base load, intermediate load, and peak hour load. Factors that affect to dispatch a specific

Table 2 Electric vehicle penetration scenarios (approximate in millions) by different organizations

\begin{tabular}{lccc}
\hline Year & US EIA - USA & NRC (probable) - USA & IEA world \\
\hline 2015 & 1 million & 1.5 million & 1.1 million \\
2020 & 2.3 million & 3 million & 6.9 million \\
2025 & 3.2 million & 7 million & 17.7 million \\
2030 & 4 million & 14 million & 33.3 million \\
\hline
\end{tabular}

US EIA: United States energy information administration [2,15]; NRC: National Research Council [2,15]; IEA: International Energy Agency [3]. 
generation asset include variable operation and maintenance $(\mathrm{O} \& \mathrm{M})$ costs, flexibility (fast vs. slow start generators), environmental 'head-room', and the distance to load and transmission. To meet the base load demand, utilities employ large scale $(\geq 400 \mathrm{MW})$ and low cost generation assets (e.g., nuclear, hydro, coal). Moreover, base load generation is characterized by high load factor (the percentage of hours that a power plant runs at full capacity) [17]. For intermediate load generation (the difference between expected customer demand and base load generation), power plants with lower load factors (typically around 50\%) such as combined cycle combustion turbine fueled by natural gas etc. are employed $[2,18]$. Finally, utilities may need to employ additional generation assets to accommodate customer demand during peak hours. For this purpose, fast start, high cost, and usually environmentally unfriendly assets are employed. They are characterized by low load factor ( $5 \%$ to $10 \%$ ), that leads to decreased utilization and hence and increased ratio of peak to average demand. Consequently, the use of such assets gradually increases the average kWh electricity price. A real-world scenario is illustrated in Figure 1a.

\subsubsection{Impact of the EV penetration}

There are a handful of studies investigating the impact of electric vehicle charging on power generation [19-23]. According to [21], plug-in hybrid electric vehicles (assuming all vehicles are PHEV20 with a battery pack of 7.2 $\mathrm{kWh}$ ) can increase the total load by $2.7 \%$ and the peak load by $2.5 \%$ in Colorado. On the other hand, battery sizes of pure EVs range from 16 to $52 \mathrm{kWh}$, which means actual impacts will be more severe. Similarly, [24] presents that if $5 \%$ of the EV population charge at the same time, there will be a $5 \mathrm{GW}$ increase in total power demand by year 2018 in VACAR region (Virginia - North Carolina - South Carolina). Overall, uncontrolled EV charging will decrease the utilization of low cost generation assets, increase the peak to average load ratio, and increase the power generation cost. Potential impacts of EV demand on the cost of the power grid is presented in Figure 1b.

\subsubsection{Opportunities}

The aforementioned effects can be mitigated with the deployment of necessary smart grid communication technologies which enable EV users to take advantage of low prices during off-peak hours. In such applications, known as valley filling, grid operators encourage customers to postpone their EV charging to low power demand periods aiming to increase the overall power grid efficiency. There are many opportunities to use valley filling applications. The US power grid uses its maximum generation only around $5 \%$ of the time [25]. If optimal valley filling programs are employed, almost $73 \%$ of the vehicles in the US can be substituted by EVs [26]. Such an approach mandates EVs to be charged during the night when the aggregated power demand is low. For instance, the authors in [27] propose an EV charging framework for valley-filling applications in New York State with varying EV market penetrations of $5 \%$ to $40 \%$. They show that the intelligent scheduling of EV chargings at off-peak hours increases the utilization of low cost generations, hence lowers the wholesale energy cost. In a similar study, authors of [23] argue that the savings gained due to intelligent charging of EVs could be reflected in charging tariffs and it promotes EV ownership. Furthermore, the work presented in [28] proposes a valley-filling algorithm and models the customer to grid interaction via pricing demand signals.

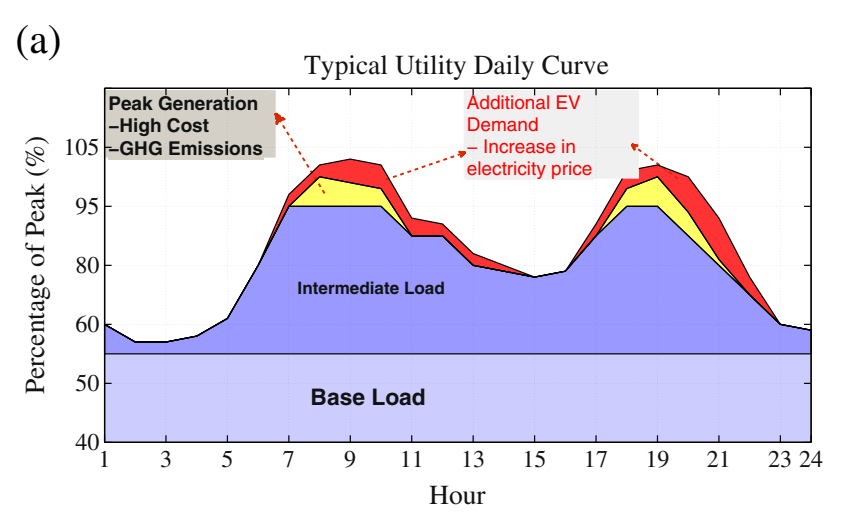

Impacts of electric vehicle penetration on power generation. (b)

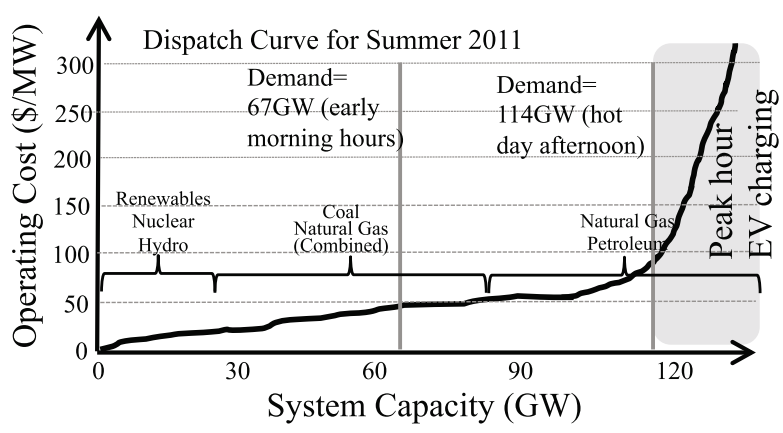

System operating cost.

Figure 1 Impacts of EV charging on power generation and system operating cost. (a) Impacts of electric vehicle penetration on power generation. (b) System operating cost. 


\subsection{Transmission network}

\subsubsection{Current status}

The transmission network ties the bulk power generation with the end users via high voltage lines. The US national grid includes three distinct geographic interconnections, namely the Eastern Interconnection, the Western Interconnection, and the Electric Reliability Council of Texas. The transmission network is composed of 170,000 miles of transmission lines rated at $200(\mathrm{kV})$ and above, delivering the power generated at 5,000 (approximately) power plants [2]. Over the last two decades, the transmission network acts as an open highway which connects wholesale electricity markets to with end users. The primary goal of the network operators, on the other hand, is to make sure that transmission lines operate efficiently and reliably as it delivers the minimum cost generation to end users.

\subsubsection{Impact of the EV penetration}

According to a study conducted by the US Department of Energy [29], in the Western Interconnection network alone, one third of the lines experienced congestion at least once during the year of the study, and $17 \%$ of the lines are congested at least $10 \%$ of the times. This study also shows that the situation is even more severe in the Eastern Interconnection, as the infrastructure is older and the network is not designed for long distance delivery of power.

On the other hand, the growth in EV load along with the deployment of new generators requires a capacity expansion in the transmission network. However, due to economical and political reasons, the required investments may not be realized in the short term. Past experiences show that new transmission projects can cost up to billions of dollars and may be stalled if the cost allocation and the recovery of investments are not properly planned. To that end, uncontrolled EV demand will allow transmission bottlenecks to emerge. These bottlenecks will increase electricity costs and the risks of blackouts.

\subsubsection{Opportunities}

The introduction of bidirectional chargers enables electric vehicles to transfer energy back to the grid (V2G) or to other electric vehicles (V2V) [30]. The utilization of such ancillary services can aid the transmission operations, mainly by reducing the congestion during peak hours. For example, group of vehicles can sell back part of their stored energy to other EVs who are in urgent need. This way, energy trading via V2V will eliminate the need to draw power from bulk power plants and hence the associated power losses in transmission will be minimized. For instance, studies in $[31,32]$ present mathematical framework to model the interaction of energy trading in a V2V scenario, where the groups of EVs determine the amount of energy to exchange and negotiate on unit price.
Moreover, EVs can transport their stored energy from one location to another which can support the grid via V2G applications. For example, [33] provides a transmission network based on the capability Internet of vehicles to transfer energy to the regions of high energy consumption. This way, the required upgrades will be deferred and occur gradually over time.

\subsection{Distribution network}

\subsubsection{Current status}

The distribution network is the final portion of the power grid which interfaces with the consumers. It is responsible for reducing the high voltage carried by transmission lines to appropriate levels for end users with the use of transformers typically rated between 2 to $40 \mathrm{kV}$. Over the last decade, the distribution network has been running up against its operating limits. In the US, national grid almost $7 \%$ of the electrical energy is lost (mostly in the form of heat) between generation units and end users and distribution network is mostly responsible for this. The distribution system is the most interruption-prone component of the power grid. According to [2], more than three-fourths of service interruptions originates in the distribution level.

\subsubsection{Impact of the EV penetration}

If charged at parking lots or customer premises, the distribution grid is the part where most electric vehicles will be attached to. Uncontrolled EV charging could stress the distribution grid and cause system failures such as transformer and line overloading deteriorate power quality (e.g., large voltage deviations, harmonics, etc.). Considering the fact that EV penetration is going to be geographically clustered, negative impacts will be more severe in certain regions $[2,34,35]$. For instance, the US distribution grid is designed to meet three to five houses [36] per transformer. Since charging of one EV doubles the daily load of a typical house, further challenges will be faced by the additional load introduced by EVs. A very typical scenario is illustrated in Figure 2 where five houses are served by a $37.5-\mathrm{kVA}$ transformer. If just two level2 chargers are used concurrently, local transformer is going to be overloaded. The frequent occurrence of such events will increase power loses and voltage deviations, and decreases transformer lifetime (high loading leads to high operating temperature) $[3,37,38]$. In [35], the authors presented a comprehensive study on the impacts of variety of EV charging scenarios on the required transformer upgrades and transformer efficiency.

\subsubsection{Opportunities}

Intelligent control mechanisms (presented in the next section) can mitigate the aforementioned effects. Such frameworks requires both parties (EVs and the grid) to 


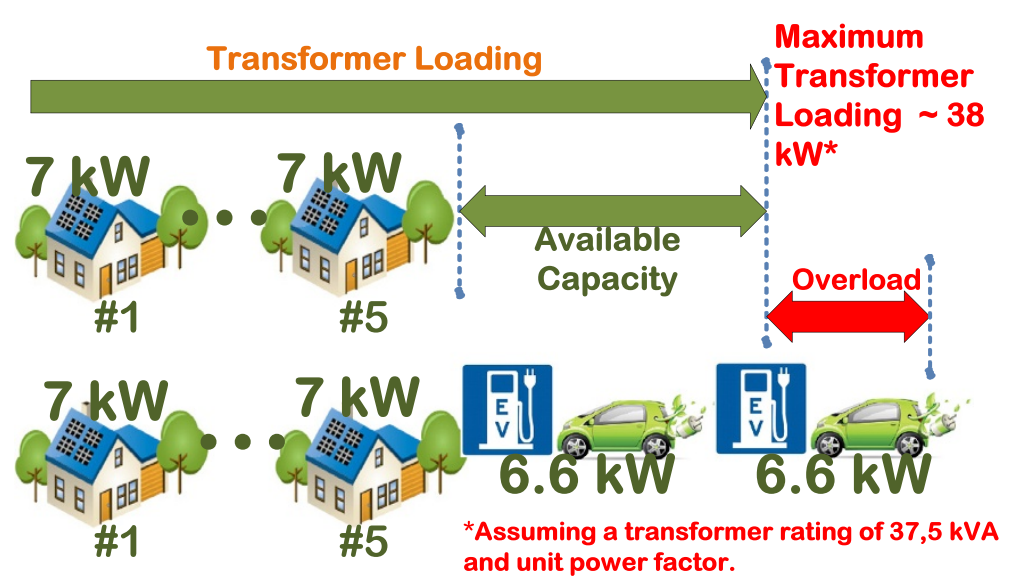

Figure 2 Potential distribution network overloading [39].

communicate. According to [40], controlling EV charging can reduce the number of congested (overloaded) network components which need to be replaced, hence eliminate the need for costly upgrades. It is further shown that controlling EV charging can reduce the cost of energy losses by $20 \%$ when compared to uncontrolled charging. In addition, EVs can be seen as distributed-energy storage mediums which are very essential for ancillary smart grid applications like integration of renewable energy resources and frequency regulation applications [41]. We provide a summary of the negative impacts of uncontrolled EV charging in Figure 3.

\section{Demand management for the internet of electric vehicles}

In order to mitigate the negative impacts of EV demand, there has been a growing interest in developing coordination strategies. At the heart of such frameworks lies information and communication technologies to support, control, and manage energy transfer between vehicles and the power grid that varies both in time and space, known as the Internet of EVs. In this section, we provide a comprehensive overview on the related literature. We classify the demand management techniques with respect to the objective of the optimization problem, scale of the problem, and the employed mathematical techniques. We present an overview of the literature in Figure 4 and the benefits of demand side management of EVs is summarized in Figure 5.

\subsection{Control objectives}

\subsubsection{Technical objectives}

The technical control objectives are usually related to the operating limits of the physical power grid assets. The most common objective functions are the minimization of energy losses, controlling voltage deviations, reducing peak-to-average load ratio, smoothing the consumer demand, and supporting renewable energy generation

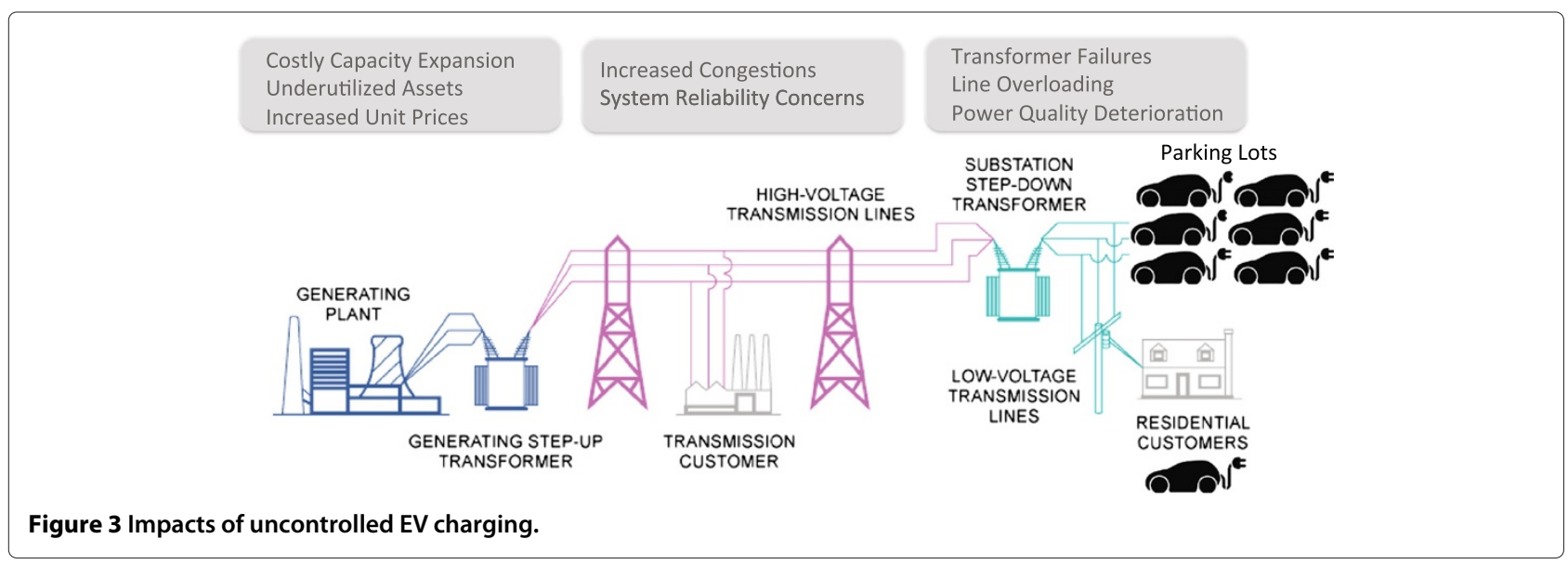




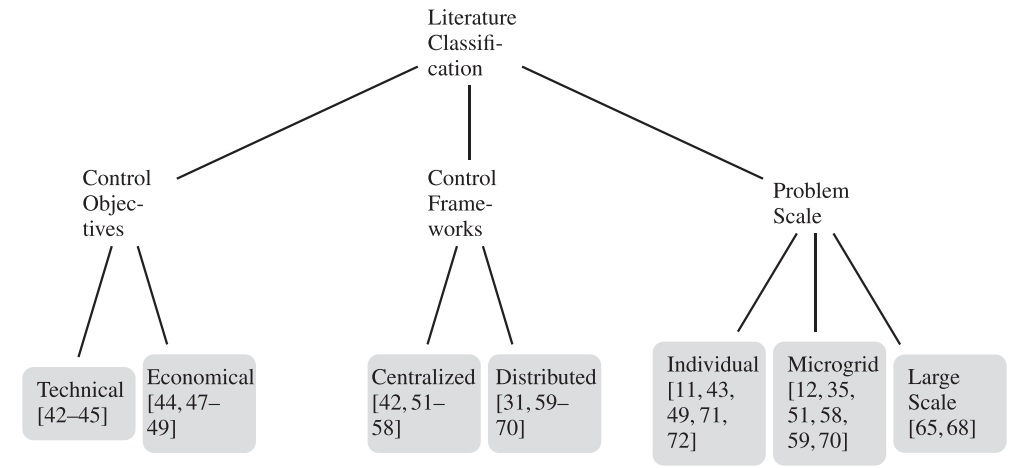

Figure 4 Literature classification of demand management techniques for loEVs.

[42-45]. For vehicle-to-grid and vehicle-to-vehicle applications, the technical objectives include battery degradations and aging, thermal stability, etc. [46].

\subsubsection{Economical objectives}

The objective functions fall into this category are usually linked to energy market participants: consumers, producers, retailers, etc. The main objectives include minimization of electricity generation and consumption costs. In this case, the objective functions are usually modeled with utility functions and the goal is to develop a charging tariff such that the total cost of charging is minimized compared to uncontrolled case [44,47-49].

It is noteworthy that both of the objective functions are actually reflected in electricity prices. Hence, in some cases, technical objectives are coupled to economical objectives. Nodal pricing can be a good example [50], where the technical aspects (distance of generators, congestion of transmission lines, etc.) are translated into cost functions and the optimal pricing is solved with a more holistic approach.

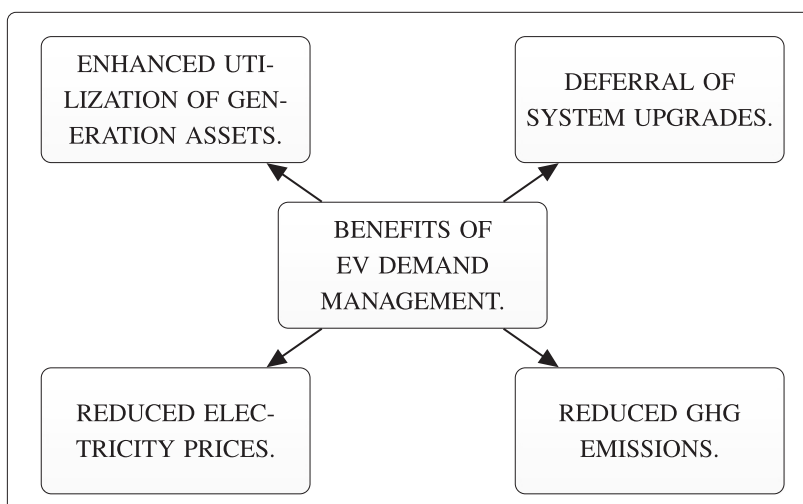

Figure 5 Benefits of electric vehicle management and control.

\subsection{Control frameworks}

The aforementioned control objectives are used in the mathematical frameworks to manage the EV demand. The applied control techniques depends on the employed charger technology. As given in Table 1, level I and level II charging typically takes a few hours, hence for these types, it is assumed that EVs are located in the customer's premises or at large parking lots. The majority of the literature considers EVs as 'smart' loads as the carving current can be adjusted in order to maximize the control objectives given above.

On the other hand, for the fast charging case, the EVs are assumed to be mobile and due to short service duration, the common control techniques include admission control at individual stations and customer routing/assignment in a network of charging stations. Overall, for both cases, the related literature can be divided into two categories: centralized and distributed controls.

\subsubsection{Centralized control}

Centralized control employs a central authority (dispatcher) who up to a large extent controls and mandates EV charging rate, start time, etc. System level decisions, such as the desired state of charge, charging intervals, etc., are taken to finish all jobs by a certain deadline (e.g., by $7 \mathrm{am}$ ). Main advantages of centralized control include higher utilization of power grid resources and real-time monitoring of operation conditions across the network. On the other hand, to enable such functionalities, an advanced communication network is needed. Studies presented in [42,51-56] are examples of centralized scheduling. These studies differ by the assumptions they make; interruptible vs. uninterruptible load, constant vs. varying charging rate, and preemptive vs. non-preemptive jobs. Management of EV fleets (e.g., school buses, postal service vehicles, etc.) can be a good example for centralized control. In this case, fleet owners can draw contracts with the utility operators and 
receive discounts. In return, utility can orchestrate EV demand according to network conditions to minimize his operating cost. Moreover, authors of [57] propose a deadline scheduling policy with admission control. They compare their algorithm with classic earliest deadline first and first come first serve scheduling. Similarly, the authors of [58] uses an admission control algorithm called Threshold Admission with Greedy Scheduling. In addition, their model incorporates renewable energy resources to charge electric vehicles.

\subsubsection{Distributed control}

Decentralized control allows customers to choose their individual charging pattern. Decisions can be based on the price of the electricity or time of the day. This method eliminates the need of third party controller (dispatcher) and complex monitoring techniques. Since decisions are taken individually, game theoretic models are extensively employed. The works presented in $[31,59,60]$ use Stackelberg game to model interactions of system operator (leader), who sets the prices and have the first move advantage, with individual EVs (followers) who respond to price changes by adjusting their demand. Another popular method is the Nash equilibrium, in which optimal pricing is achieved through maximization of individual utility functions [61,62]. Other employed models include mean field games, potential games, and network routing games [61-70]. In addition to scheduling of night time charging, there is an interest in large scale charging of group stationary EVs (park and charge). For instance, [71] uses swarm optimization to allocate power to EVs in a parking lot. Authors of [72] propose a combined pricing-scheduling quadratic integer programming model to determine optimal prices and schedules to manage EV demand in large scale parking lots.

\subsection{Scale of the problem}

The scale of the control framework can vary from individual level to entire transmission voltage level. We classify the scale of the problem into three categories.

- EV scale: This level of scale considers coordination of individual EVs according to the available information at the customer premises. Economical goals such as cost minimization and load profile smoothing are usually chosen $[43,49,59,73]$.

- Microgrid scale: This level of problem considers groups of vehicles connected to LV/HV feeders. Typical examples include university campuses, parking lot (malls, airports, etc.), and microgrids. The control and coordination studies at this level include $[12,34,58,71]$.
- Transmission scale: At this scale transmission, system operators and wholesale energy markets operate. Corollary, the control techniques applied considers thousands of EVs located in large geographical regions. The primary goal of this scale is to develop pricing policies to achieve optimal valley-filling during night time [62,69].

\section{Available communication standards and technologies}

The IoEV is based on the information and communication infrastructure to support the control and manage the energy transfer between vehicles and the power grid. In order to support such frameworks, we survey the related technologies and standards and the interdependency diagram which is presented in Figure 6. As this is a new area, some of the standards are either published or under development. We classify the communication standards and technologies into three groups: (1) the first group includes the technologies that are responsible for home charging applications and the message exchange between the EV and the charging equipment; (2) the second group includes the technologies for the mobile EV communication; and (3) the third group includes the standards for 'inter-control center' communication.

\subsection{Communication needs at customer premises \\ 4.1.1 EV-electric vehicle supply equipment}

The communication at customer premises takes place in several places. First, group contains the standards and technologies between electric vehicle and electric vehicle supply equipment (EVSE) that is required for energy transfer monitoring and management, billing information, and authorization. The standardization is required for fast adoption of EVs and proper functioning of electric vehicle network components. The Society of Automotive Engineers (SAE) have defined the communication standards when an EV is being charged. We described these standards below $[74,75]$.

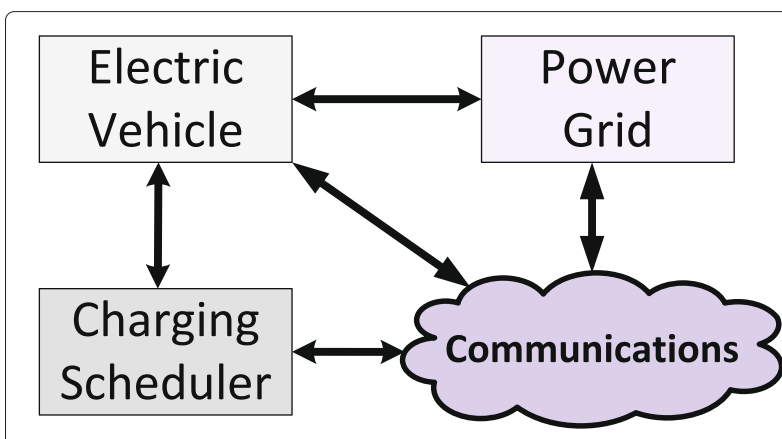

Figure 6 Interdependency of communications and EV demand management. 
- SAE J2293: This standard covers the functionalities and architectures required for EV energy transfer system.

- SAE J2836/1 and J2847/1: Define use cases and requirements for communications between EVs and the power grid, primarily for energy transfer. The central focus is on grid-optimized energy transfer for EVs to guarantee that drivers have enough energy while minimizing the reducing the stress on the grid.

- SAE J2836/2 and J2847/2: Define the uses cases and requirements for the communications between electric vehicles and off-board DC charger.

- SAE J2836/3 and J2847/3: Identify use cases and additional messages energy (DC) transfer from grid to electric vehicle. Also supports requirements for grid-to-vehicle energy transfer.

- SAE J2931: Defines digital communications requirements between EV and off-board device. SAE J2931/1 covers power line communications for EVs.

- SAE J2931/2: Defines the requirements for physical layer communications with in-band signaling between EV and EVSE.

In Figure 7, an overview of SAE communication standards is presented. For instance, J2836/1 use cases for utility programs may include time of use program, real-time pricing program, or critical peak pricing program [76]. Moreover, the International Electrotechnical Commission (IEC) is developing several standards under development for DC fast charging option. IEC 61851-23 presents the requirements for gird connections and communication architecture for fast charging. IEC 61851-24 defines the digital communications between $\mathrm{EV}$ and EVSE.

\subsubsection{Energy management unit to power grid}

Visualization of energy consumption clearly helps customers to understand the cost of their energy usage. However, optimal decisions can only be taken by automated management systems [77,78]. Energy management units (EMU) enables customers to power grid interaction;

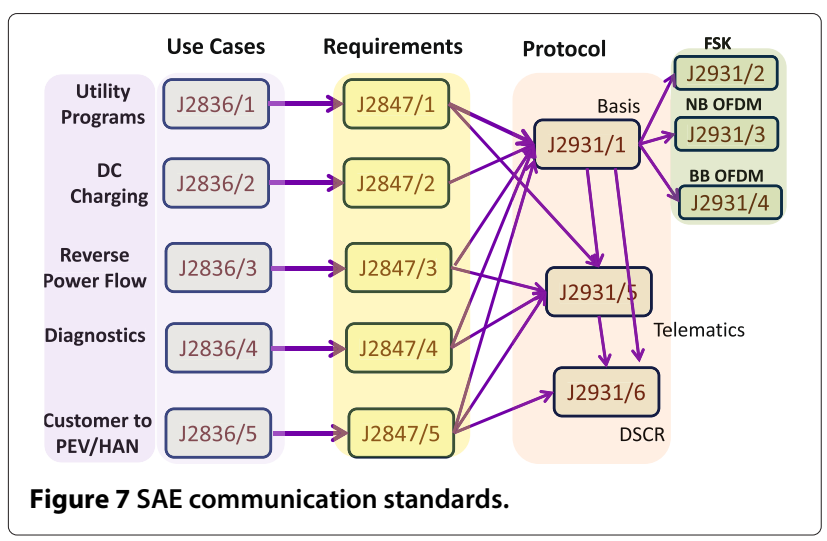

customers can monitor, control, and optimize their energy consumption. Even though energy management systems have been in the market for a few decades, the widespread adoption has gained pace with the recent advances in smart grid. [77] presents recent advances in EMUs.

EVSE will connect to EMU via home area network (HAN). The most popular technologies for HAN are Zigbee [79,80], 802.11-based wireless local area network (WLAN), and femtocells. Zigbee offers required coverage (30 to $40 \mathrm{~m}$ ), data rate (256 Kbps), low power usage, and deployment cost. In fact, it has a considerable market share in utility world $[7,8]$. The ubiquity of 802.11 -capable devices makes WLAN a strong candidate for HAN. The details of WLAN technology is given in the next section. A comprehensive summary is presented in Table 3.

Femtocells are usually employed as access points of cellular networks. This technology uses customer's broadband, DSL, etc. to connect to the wireless carrier's core network. This way, femtocells offer required indoor coverage and capacity for smart grid applications. Communication technologies with a special focus on security for home area networks is presented in [81].

For residential charging, the communications between EMU and the power grid is supported by the existing advanced metering infrastructure (AMI) network [82]. There are several candidates for this purpose.

Power line communications (PLC): PLC is a strong candidate for EMU to grid interaction. The main motivation for PLC is that already existing grid infrastructure reaches every EMU that wants to charge an EV. There are three different types of PLC technologies which are classified by the used frequency band and data rate. Broadband PLC

\section{Table 3 Summary of candidate wireless technologies for IoEVs}

\begin{tabular}{lcccc}
\hline & Latency & Throughput & Security & Scalability \\
\hline WiFi & & & & \\
IEEE 802.11a & $L$ & $\mathrm{M}$ & $\mathrm{M}$ & $\mathrm{M}$ \\
IEEE 802.11b & $\mathrm{L}$ & $\mathrm{H}$ & $\mathrm{M}$ & $\mathrm{L}$ \\
IEEE 802.11g & $\mathrm{L}$ & $\mathrm{H}$ & $\mathrm{M}$ & $\mathrm{M}$ \\
IEEE 802.11n & $\mathrm{L}$ & & & \\
3G & & $\mathrm{M}$ & $\mathrm{H}$ & $\mathrm{H}$ \\
UMTS/HSPDA & $\mathrm{M}$ & $\mathrm{M}$ & $\mathrm{L}$ & $\mathrm{H}$ \\
EVDO & $\mathrm{M}$ & & & $\mathrm{H}$ \\
4G & & $\mathrm{H}$ & $\mathrm{H}$ & $\mathrm{H}$ \\
LTE/HSPA+ & $\mathrm{L}$ & $\mathrm{H}$ & $\mathrm{H}$ & $\mathrm{H}$ \\
IEEE 802.16e & $\mathrm{L}$ & $\mathrm{H}$ & & \\
\hline
\end{tabular}

Wireless mesh network can be implemented with WiFi nodes. Low (L): latency $(<250 \mathrm{~ms})$, throughput $(<500 \mathrm{Kbps})$, scalability $(<100$ nodes/backhaul node). Medium (M): latency ( $250 \mathrm{~ms}$ to $1 \mathrm{~s}$ ), throughput (500 to 1,500 Kbps), scalability $(<100$ to 1,000 nodes/backhaul node). High $(H)$ : latency ( $>1 \mathrm{~s})$, throughput (> 1,500 Kbps), scalability (> 1,000 nodes/backhaul node). 
uses 1.8 to $250 \mathrm{MHz}$ frequency band and physical data rate varies between a few megabits to hundreds of megabits. Narrowband PLC operates in the 3 to $500 \mathrm{kHz}$ band and provides lower data rates. Third type of PLC communications is ultra narrow band technology, which is also the oldest type of all three. It only provides data rate around hundred bits per second [83].

Several millions of PLC-based communications have already been deployed globally [84]. Moreover, for EV to EVSE communications, PLC supports an apparent physical association that cannot be achieved by its wireless alternatives. Another distinctive advantage is that the cost of PLC deployment is relatively low when compared to other wireline options and can be comparable to wireless technologies.

However, there are several disadvantages for PLC. First, the communication medium is harsh and noisy. Second, transformers cause high attenuation which limits the range of the communication. Repeaters can be employed to overcome this problem, but additional cost should be taken into account beforehand. The final disadvantage is that regulations in some countries limits the use of PLC. For instance, PLC is not allowed for indoor environments in Japan [85].

White-space networking: The long term assignment of wireless spectrum to parties like digital TV broadcasters has created inefficient use of ISM band. Fatemieh, 2010 [86] proposes to use TV white spaces to meet communication requirements between users and the grid. IEEE 802.22 is the wireless regional area network (WRAN) standard that uses white spaces in the spectrum. The use of this technology offers the following benefits. It allows high data rates in a cost-effective way. White space networking has deep penetration and long range transmission capabilities, which would eliminate the need for complex designs (for EMU to data aggregation units). Also, high coverage can easily be achieved using white spaces. IEEE 802.11af, also referred to as 'White-Fi' and 'Super Wi-Fi' is a recent proposal that allow WLAN operation in TV white space spectrum in the VHF and UHF bands $[87,88]$. It uses cognitive radio technology to transmit on unused TV channels, with the standard taking measures to limit interference for primary users, such as analog TV, digital TV, and wireless microphones.

However, white-space networking is challenging. Available white spaces must be detected and interferences with the incumbents should be avoided. The underlying network should be able to run for varying bandwidths. Also, there are issues related to operation and management of the network $[85,86]$.

Wired infrastructure: Another option might be to build a wired infrastructure. Dedicated communication links give utilities full control over the network and reduce the reliance on the communication infrastructures operated by third parties. However, building such wired infrastructures is very costly. On the other hand, if the two-way communications is going to be a part of the power grid for the next century, it might be logical to build such an infrastructure gradually over time.

Customer's broadband: One school of thought suggests to use commodity broadband technologies, e.g., digital subscriber lines (DSL) or cable. The capital expenditures (CAPEX) for this case are lower, as the main communication infrastructure has already been deployed. Moreover, commodity broadband technologies uses Internet protocol (IP), so it can be easily connected to other ubiquitous IP-based communication networks. In a recent deployment, a DSL network was used as an underlying communication technology in Boulder, Colorado [89]. Nonetheless, there are several handicaps. The number of broadband connections is lower than the number of power meters. This is especially the case in developing countries. Moreover, the down times in some deployments is unacceptable for critical smart grid applications.

Other technologies: Mesh networks [85] have been proposed as alternative communication technology for AMI networks. Mesh networks tend to use different forms of wireless networks, i.e., IEEE 802.11, 3G/4G/5G, and mesh type of radio configuration. This choice is subject to technical, strategic, and even legal constraints. We present a detailed overview of such technologies in the next sections. In Table 4, we present an overview of candidate technologies and network technologies such as 3G/GSM, 4G/LTE (via smart apps such as $[90,91]$ ).

An overview of the communication technologies for garage charging is presented in Figure 8 and summarized in Tables 5 and 6 . Note that the communication requirements for the EV to EVSE is in the orders of milliseconds, while EVSE to EMU communication can occur in the order of seconds. Finally, the EMU can communicate with the grid in the order of minutes (typically every $15 \mathrm{~min}$ ). In the next section, we will provide a comprehensive overview of such communication requirements.

\subsection{Mobile EV to control center communications}

Mobile EVs use public fast charging stations to fill up their batteries. Customer demand varies both spatially and temporally (e.g., downtown areas during rush hours) [37]. Also, the current status of the power grid limits grid operators to deploy the required number of charging stations. Hence, customer demand should be balanced among neighboring stations through the use of communication infrastructures. Thus, the ability to share data for mobile EVs becomes a necessity. In Figure 9, we present an overview of message exchange in electric vehicle networks.

There are several wireless communication technologies that are projected to support 'electric mobility'. Two 
Table 4 Candidate communication technologies customer-to-grid interaction for garage charging

\begin{tabular}{lll}
\hline Technology & Pros & Cons \\
\hline Power line communications & Every EV owner has an access. Easy penetration & $\begin{array}{l}\text { Indoor applications are not allowed in every country. } \\
\text { Regulatory and technical issues }\end{array}$ \\
$\begin{array}{ll}\text { White-space networking } \\
\text { Utility-owned wired infrastructure }\end{array}$ & $\begin{array}{l}\text { High penetration and coverage } \\
\text { interoperability among various standards }\end{array}$ & Require technologies to operate at varying bandwidths \\
Fixed broadband & Low cost (customers already have it) & Level of broadband deployment can be problematic \\
Wireless cellular networks & Easy adoption with already existing structure & Coverage is limited in developing countries \\
WiFi mesh network & Low cost, unlicensed frequency band & May require complex designs
\end{tabular}

strong candidates are cellular network communications and wireless mesh networks.

\subsubsection{Cellular network communications}

For the short term, ubiquitous public cellular networks can provide required communication coverage in a costeffective way. Moreover, cellular operators offer service solutions for smart grid applications. Power meter manufacturers embed communication modules to enable use of cellular communications. For garage charging and vehicleto-grid applications data (e.g., power usage, price, etc.) are exchanged periodically (typically around every 10 to 15 min). Most cellular networks have sufficient capabilities to support the required communication medium. Further, cellular networks have the following advantages: (1) cellular communication technology is mature enough to meet smart grid needs; (2) since all cellular networks operate on licensed spectrum, there is no need to pay for unlicensed bands; and (3) cellular networks are scalable enough to connect huge number of EVs.

Worldwide interoperability for microwave access (WiMAX) is another candidate. WiMAX offers high capacity, wide coverage, low latency, low per-bit cost, and required quality of service capabilities. For example, garage charging applications generate small amount of traffic, but the projected number of connections is very high. For mobile EVs, high data rate is needed to support location based applications. In most cases, in-vehicle application requires wide coverage, high throughput, and QoS support. WiMAX has required capabilities to handle the transmission of such data. In addition, mobile data service based on 4G long term evaluation (LTE)

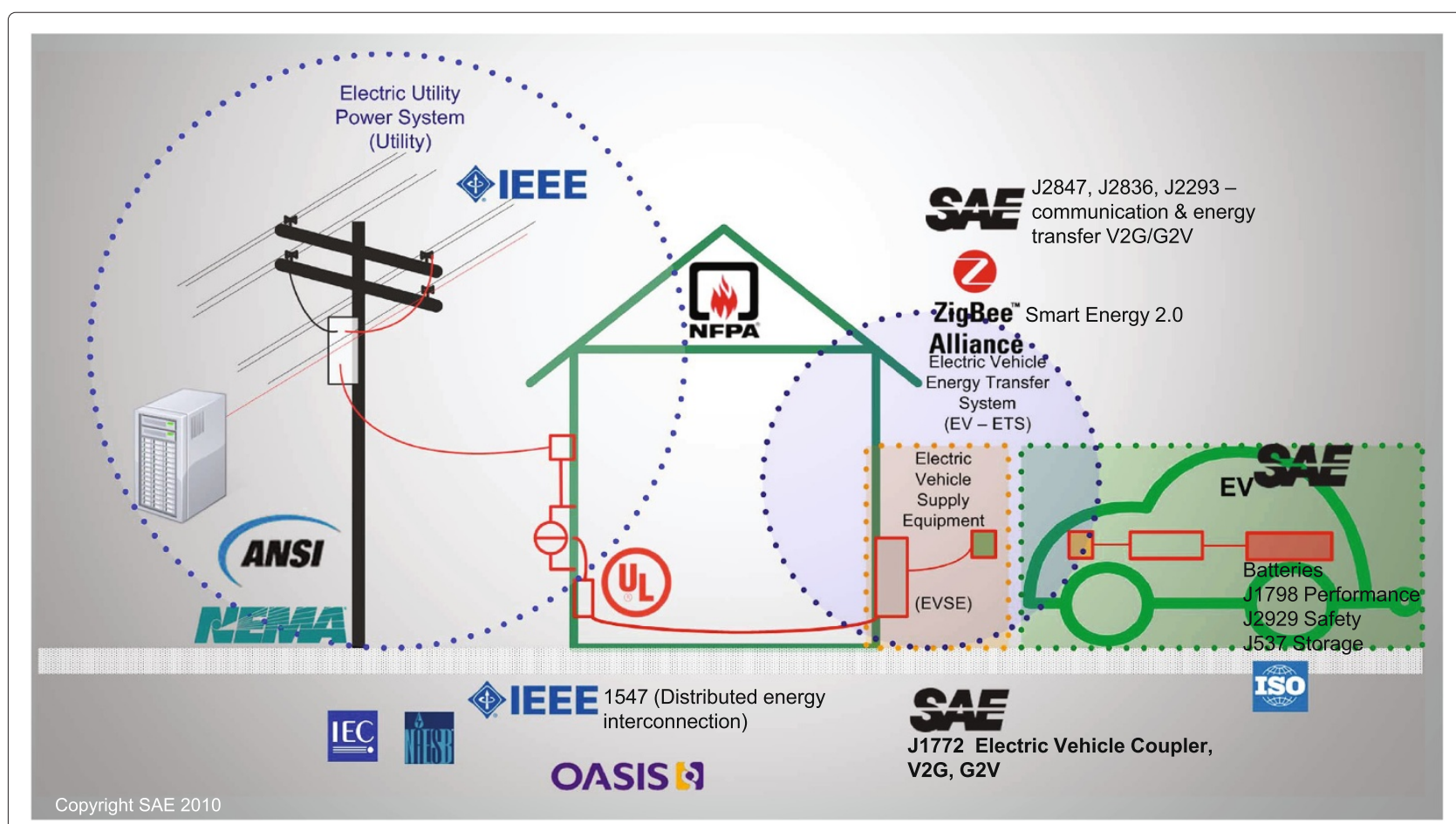

Figure 8 Overview of electric vehicle energy transfer standards (used with permission of SAE International [92]). 
Table 5 Overview of communication standards for loEVs

\begin{tabular}{lll}
\hline End users & Application & Name of standards and technologies \\
\hline EV-EVSE & Energy transfer - garage charging & SAE J2293, SAE J2836/1, J2847/1, SAE J2836/2, J2847/2, \\
& & SAE J2836/3, SAE J2847/3, SAE J2836/4, J2847/4, SAE J2931, \\
IEC 61851-23, IEC 61851-24 \\
EVSE - Energy Management Unit (EMU) & Home area network & Zigbee, 802.11, HomePlug \\
Customer (EMU) - grid & $\begin{array}{l}\text { Garage charging, load shifting, } \\
\text { valley filling, energy trading }\end{array}$ & PLC, 3G/4G/WiMAX/LTE/5G, WMN, TV white space, DSL, cable \\
Mobile EV - control center & Public charging & 3G/4G/WiMAX/LTE/5G, WMN \\
Inter-control center & Public charging & IEC 60870-6/TASE.2 \\
\hline
\end{tabular}

is becoming more popular as it can provides browsing experience comparable to wired connections. As of August 2013, there are more than 176 million LTE customers exist in the globe, and this number is expected to grow exponentially and exceed 1.3 billion by the end of 2018 [93]. Hence, 4G/LTE can provide a ubiquitous communication for EVs.

On the other hand, public charging applications require mobility support. As the mobile user moves faster, the supported data rate decreases. In Figure 10, we compare wireless communication networks according to mobility and throughput. 2.5G, 3G, 4G (WiMAX and LTE), and the upcoming $5 \mathrm{G}$ offer required connectivity for mobile EVs. IEEE P2030 standard [94] presents possible communication interfaces. The connection to central controller or telematics provider can be established by either equipment manufacturers OEMS or wide area communication.

\subsubsection{Wireless mesh networks}

Wireless mesh networks (WMNs) are qualified to deliver required connectivity to EV drivers and the power grid. Moreover, their low cost, high scalability, self-healing, and self-organizing nature along with mobility support makes WMNs a very strong candidate. WMNs can provide high bandwidth and seamless handover capabilities at high speeds (almost the same quality as third generation technologies) [95]. Also, WMNs are compatible with other networks: they can be integrated with other existing networks (e.g., IEEE 802.15, IEEE 802.16, cellular networks, etc.). Further advantages of WMNs include its higher physical layer transmission rate than most cellular networks and coverage can be extended without using extra channel capacity.

Several companies already deployed WMNs for smart grid applications [96,97]. As EV population continue to grow fast, the need for a dedicated communication infrastructure will become more important. Especially in urban environments, where ' $x G$ ' networks are overloaded or not deployed yet, WMNs will become even more important. In [97], a medium city is successfully deployed with wireless mesh networks to support required connectivity to electric vehicles.

On the other hand, WMNs have several disadvantages. In urban environments, network coverage can be affected by interference and fading. Available bandwidth can reduce in the case of possible loop problems [8]. In order to enjoy benefits of WMNs, research efforts are being shown to solve complexity of these networks.

\subsection{Inter-control center communications}

As shown in Figure 9, different regions are served by different service providers. Each control center monitors and controls registered customer demand at each charging facilities connected to him. Moreover, when a

Table 6 Summary of findings: communications needs and requirements for loEVs

\begin{tabular}{|c|c|c|c|c|}
\hline \multirow[t]{2}{*}{ Application } & \multicolumn{2}{|c|}{ EV perspective } & \multicolumn{2}{|c|}{ Grid perspective } \\
\hline & Communication needs & $\begin{array}{l}\text { Communication } \\
\text { requirements }\end{array}$ & Communication needs & $\begin{array}{l}\text { Communication } \\
\text { requirements }\end{array}$ \\
\hline Public charging & $\begin{array}{l}\text { Locate and reserve } \\
\text { charging station }\end{array}$ & $\begin{array}{l}\text { High availability, } \\
\text { service differentiation } \\
\text { may be required }\end{array}$ & $\begin{array}{l}\text { Load balancing among } \\
\text { neighboring stations }\end{array}$ & $\begin{array}{l}\text { QoS requirements } \\
\text { increases with EV } \\
\text { population }\end{array}$ \\
\hline Residential charging & $\begin{array}{l}\text { Respond to price updates } \\
\text { to minimize charging cost }\end{array}$ & $\begin{array}{l}\text { Part of AMI network } \\
\text { (see [85]) }\end{array}$ & $\begin{array}{l}\text { Valley filling to better } \\
\text { utilize power generation }\end{array}$ & $\begin{array}{l}\text { Price updates sent } \\
\text { every } 15 \text { min. } \\
\text { Requirements for AMl } \\
\text { hold }\end{array}$ \\
\hline Energy trading via V2G & $\begin{array}{l}\text { Sell part of stored energy } \\
\text { to make profit or use } \\
\text { stored energy during peak } \\
\text { hours }\end{array}$ & $\begin{array}{l}\text { High security and } \\
\text { availability }\end{array}$ & $\begin{array}{l}\text { Decrease the volume of } \\
\text { storage medium needed } \\
\text { by purchasing energy } \\
\text { from EV fleets }\end{array}$ & $\begin{array}{l}\text { The same as EV } \\
\text { perspective }\end{array}$ \\
\hline
\end{tabular}




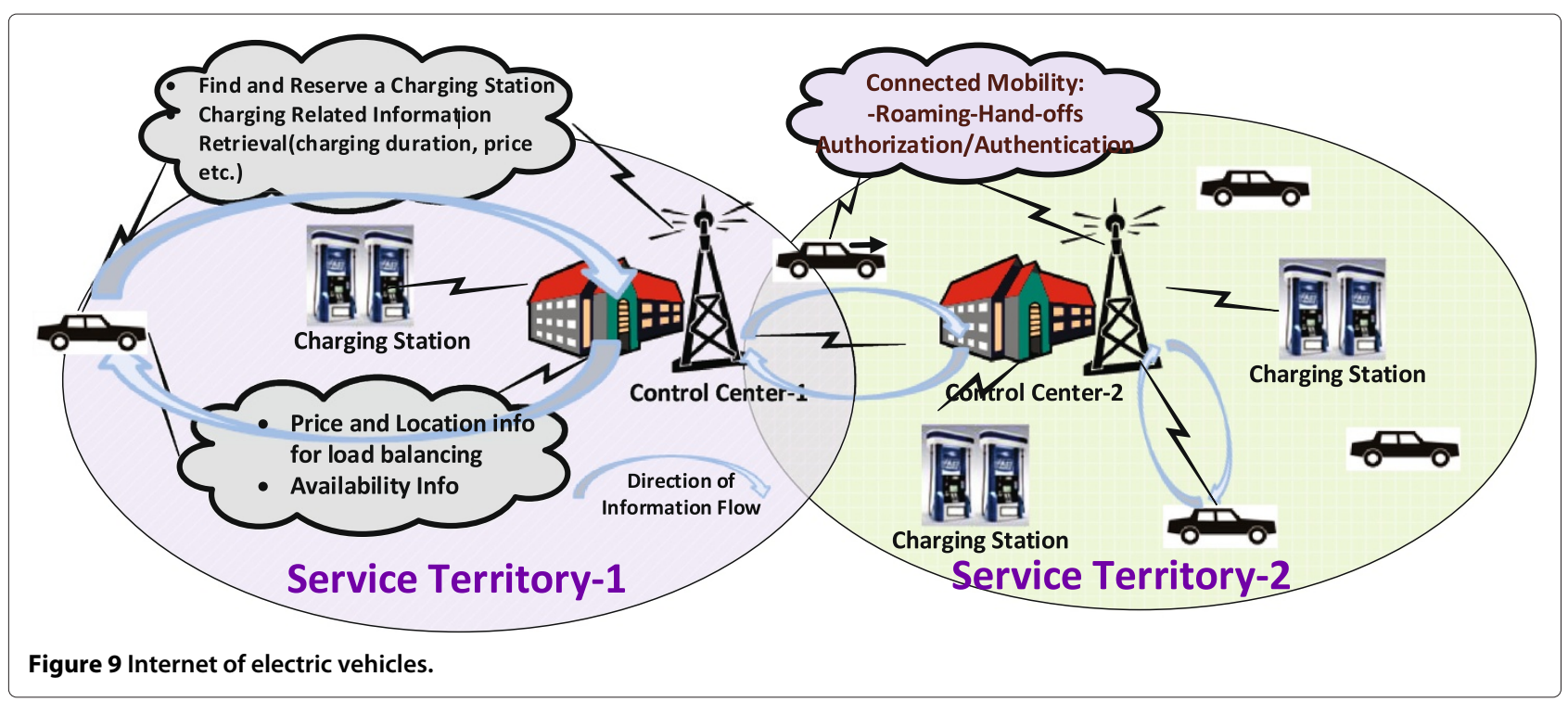

customer from another service territory requests service, control centers should be able to exchange information for authentication, billing, and location. Currently, all-electric range of most EVs is more than hundred miles [2]. This range enables drivers to go to different regions that are served by some other utility (e.g., Central Europe etc.). Hence, the communication network should be able handle possible hand-off situations.

At the present time, utilities employ IEC 608706/TASE.2 (International Electrotechnical Commission Tele-control Application Service Element) communication standard for information exchange between control centers, utilities, and power pools [8]. However, additional communication features may be needed to keep track of mobile users.

\subsection{Further communication needs}

Further, communication needs exist between EV and the charging equipment for the following periods: precharging, during the charging, and post-charging. In order to start the charging process, the EV and the charging equipment must be physically associated. Additional messages should be exchanged for identification and authorization purposes. During the charging, several parameters such as charging duration, direction of energy flow, available power and energy rate, vehicle status information (e.g., battery state of charge, usable battery energy, etc.) are needed to be exchanged between EV and EVSE. Precise measurement of transferred energy is also important for billing purposes [94].

\section{Communication requirements and performance metrics}

The end-to-end communication requirements for EV network applications require highly available, reliable, and secure communications. Different applications, such as V2G, load shedding, etc., may have different communication requirements. The use cases for EV applications serve as a starting point for communication requirements. A detailed use case analysis is presented in $[98,99]$. Each

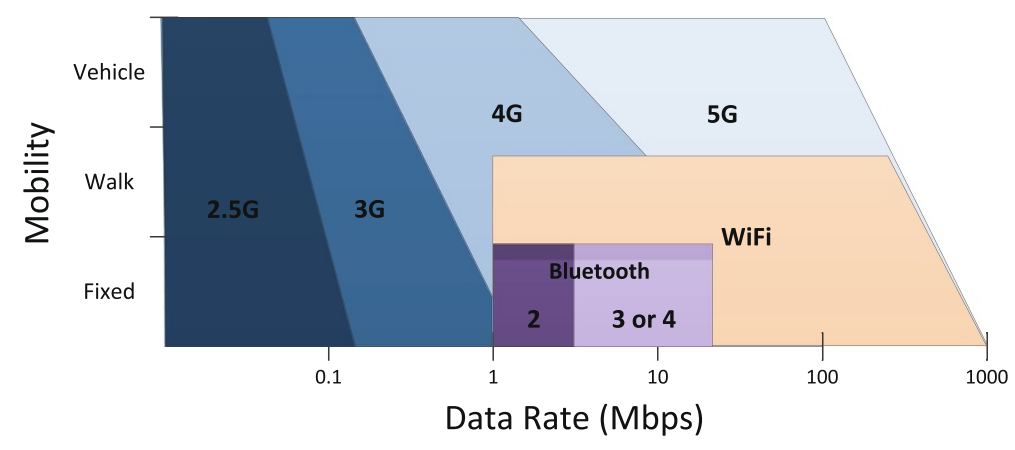

Figure 10 Data rate vs. mobility. 
use case scenario defines the end-users (e.g., customer, utility, EV, etc.), their types (e.g., individual, organization etc.), content, size, and the frequency of the required message exchange. In this section, we discuss communication system requirements and associated performance metrics.

\subsection{System reliability and availability}

The successful management of EVs requires extensive use of reliable and (highly) available IoEV. The loss of availability is going to terminate the grid to customer interaction. During these isolation periods, customers will not be able to receive electricity prices, hence cannot optimally adjust and schedule their electricity usage. In fact, the cost of unavailability can be more severe. For instance, for garage charging scenarios, uncontrolled EV charging may lead to unwanted peaks and may overload some of the grid components, such as the distribution transformer.

Considering the aforementioned use cases, [100] explores the reliability requirements for home charging EV applications. The authors show that 11 different messages are used, and the minimum reliability requirement varies between $98.8 \%$ to $99.5 \%$. This variety is attributed to some messages, such as vehicle identification number (VIN) information request, error messages related to EV charging rate, require high availability than other types.

The connectivity loss for mobile EVs is even more critical. Unavailability will refrain customers from locating and scheduling charging stations. Similarly, it may lead to suboptimal station selection both for customers (more expensive) and the grid operator (busy stations or long waiting lines may cause customer dissatisfaction) $[101,102]$. There are a handful of studies that quantify the cost of bad communication system performance. For instance, garage charging applications use AMI network. In a related study, [103] presents a generic AMI communication network and performs availability analysis for each component (e.g., home area network, 3G network, etc.). Moreover, it quantifies the cost of unavailability due to suboptimal power allocation.

There exist quite a few studies that present the performance evaluation of related wireless communication technology (e.g., UMTS etc.) [104-106]. A similar approach can be applied to mobile EV networks to quantify the cost of suboptimal charging station selections. On the other hand, redundancy design may help to improve system reliability. Employing redundant communication links between critical nodes such as data aggregation units to utility or between control centers. We present the overall system in Figure 11.

\subsection{Quality-of-service}

The quality-of-service (QoS) needs are gradually increasing as the EVs gain widespread acceptance. Since centralized or decentralized control of EVs is done via price signals, degradation in communication system performance may cost. In [108], authors define QoS requirements for general smart grid communications using in terms of communication delays and outage probability.

The QoS requirements can be slightly different for mobile EVs and the grid operator. For instance, IEEE P2030 [94] states that an EV can afford to have a few seconds of latency to retrieve location, pricing, and availability information. However, in order to respond to the huge number of queries (approximate number depends on the EV penetration level) grid operator have to receive the information in a timely manner.

Even though today's mobile broadband technologies (e.g., 3G/HSPA/EV-DO etc.) promise high throughput and low latency communications, in some occasions, there can be a degradation in the user experience. This is attributed to the network capacity saturation in some areas. For instance, [109] shows that customer demand is going to exceed network capacity, for most metropolitan areas, in the next years. This will force time critical data transfer from EVs to compete with other bandwidth

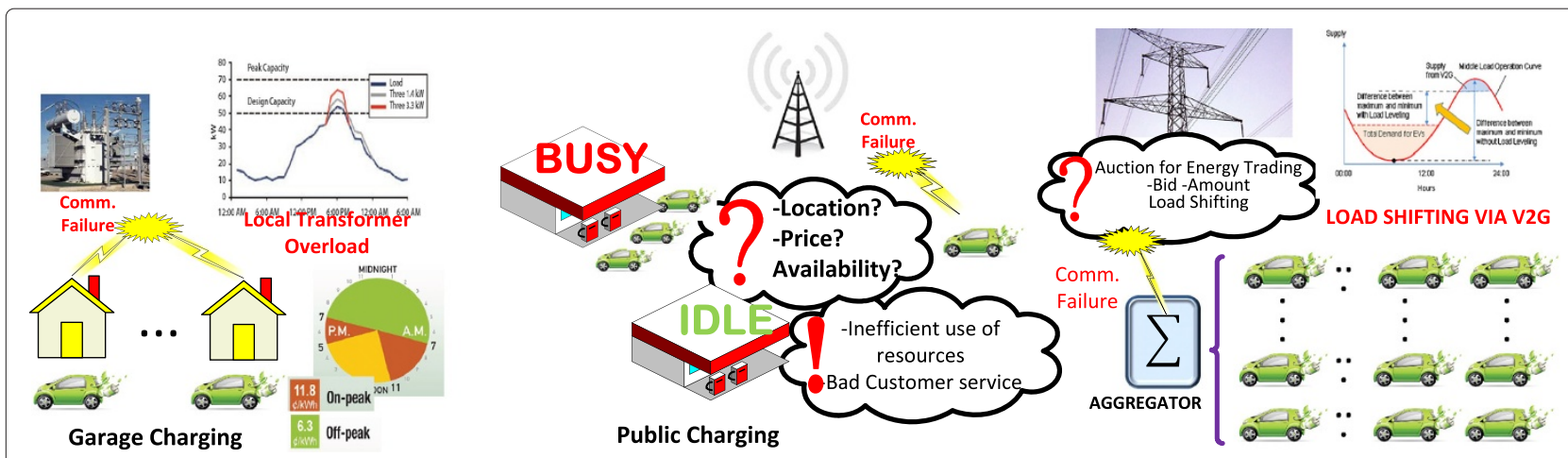

Figure 11 The negative effects of communication unavailability. Left panel: uncontrolled charging [2], middle panel: suboptimal charging station selection, and right panel: unable to support required storage medium for load shifting [107]. 
demanding applications such as video streaming and voice over IP.

On the other hand, the most recent mobile WiMAX/LTE technology can support necessary QoS requirements. More specifically, WiMAX offers four different QoS level, namely [110,111] (1) unsolicited grant service (UGS); (2) real-time polling service (rtPS); (3) non-real time polling service (nrtPS); and (4) best effort (BE). UGS can support low latency and low jitter and prioritize EV charging related data transfer. However, 4G technologies are not available everywhere and a limited but growing number of devices support 4G connectivity. Finally, some discussion is already undergoing about new 5G technologies [112].

In some areas, wireless mesh networks have been deployed using different versions of the IEEE 802.11 protocol. The cost of building such infrastructure is not expensive and does not require permission, since they function in the open $2.4 \mathrm{GHz}$ or $5 \mathrm{GHz}$ band. These networks can provide application access priority (starting from 802.11e and more recently with the 802.11ac), but they do not guarantee any strict QoS [113-116]. In addition, they have a limited range, which means that vehicles that want to communicate through them may be in a wireless blind spots.

\subsection{Cyber-physical security}

The power grid is vital to human life and with the integration of information systems, the power grid becomes a huge cyber-physical system. The grid's unique nature poses new series of security challenges. The components of the power grid are vulnerable to a variety of new cybersecurity threats that could affect national security, pubic safety, and revenues.

There has been an increasing interest in smart grid security aspect [117-126]. In [120], the authors present cyberphysical security overview of smart grid communication infrastructure. Su, 2012 [119] presents security threats for electric vehicle networks. They conclude that electric vehicle networks have the following security requirements: (1) availability (discussed in the previous section); (2) confidentiality (prevent attackers to obtain private information); (3) integrity (block unauthorized users from changing the data); and (4) authenticity.

If the security of the EV network communication is not provided at a high level, an adversary can impact the EV network in various ways. A hacker can route customers to a specific charging station to create chaos for drivers. Similar to a home appliance, the garage charging is also programmed to fill up EV battery when price is low. An adversary can launch an attack to inject negative prices to increase power usage (of automated appliances), which may result in a peak or spike in electricity usage. Similarly, price modification can cause instabilities in V2G energy trading.

In [126], the authors present the security threats in physical layer of wireless communications for smart grid applications. Moreover, [125] defines the attack types for smart grid communication networks. They introduce three different kinds of smart grid attacks:

- Data injection: The type of attacks in this category falsify the meter measurements (e.g., garage charging) to mislead the power grid operator. The main purpose of this type of attack is to create revenue loss.

- Vulnerability: This type of attack is caused by the failure of a communication channel or a device. Information on the feedback channel can be unsynchronized due to erroneous communication links.

- Intentional: In this type, the attacker has the full knowledge of network topology. It can be carried out by targeting the node with the highest degree with a denial-of-service attack.

Several organizations including IEEE (1402-2000, IEEE Guide for Power Substation Physical and Electronic Security), North American Electrical Reliability Corporation Critical Infrastructure Protection (NERC-CIP), National Infrastructure Protection Plan (NIPP), and National Institute of Standards and Technology (NIST) [118]. In the second volume of NISTIR 7628 [122], NIST documents a comprehensive overview of guidelines for smart grid cyber-security. This documents contains several use cases concerning the security issues with EV charging. In [124], the authors evaluated the effectiveness of NISTIR framework for an electric vehicle charging infrastructure case. They claim that NISTIR 7628 framework is not strong enough in device authentication and protecting the protecting the location privacy of mobile EVs.

\subsection{Scalability}

As the EV population is continuously going to increase for the next couple of decades, the underlying communication networks should be scalable enough to support required functionalities. Such scalability concerns can be alleviated by employing IP-based network designs. Considering the big smart grid picture on mind, it is very likely that that required communication networks will be based on IPv6. Moreover, IP-based solutions offer huge cost savings in deployment and maintenance [7].

\subsection{Capacity}

Since EV applications generate data traffic, the underlying communication networks should be have enough capacity to meet minimum communication requirements. For 
mobile EVs, the required capacity can be measured in bits-per-second. However, for residential charging applications, the communication capacity is more likely to be measured in the maximum number of advanced meters (or smart meter) that it can support at a time (since most messages types/lengths are standard).

In a related study [127], researchers analyze the capacity of a linear chain network topology for an AMI network. They also compute the required network capacity for different amounts of nodes, varying message lengths, and meter reading periods (e.g., every 10 or $15 \mathrm{~min}$ ). They also extend their study for larger networks with different communication infrastructures.

On the other hand, capacity comes at the expense of cost. Capacity planning is a critical step as it includes trade-offs that could affect the success of EV applications. Initial deployments may seem easy and does not require high capacity networks, since EV population will be low. This will allow utilities to have a good head start with low installation cost. However, short term solutions are likely to fail to scale. Hence, the expected exponential growth in EV population may force utilities to replace the entire communication network.

\subsection{Interoperability}

The proper functioning of EV-related applications depends on different entities such as power system and communication system to work together. According to the US Independence and Security Act (2007), the NIST is appointed to be the main global coordination of such smart grid interoperability.

In its framework [128], NIST identifies the domains of the smart grid as: customers, markets, service providers, operations, bulk generation, transmission, and distribution. NIST's conceptual framework also provides the required information exchange between these domains. EV applications are unique in the sense that they bridge most of these domains. For instance, home charging deals with distribution network and the service provider, V2G deals with markets, and public fast charging is related to bulk transmission and customers.

IEEE P2030 Smart Grid Interoperability Series of Standards aims to establish an interoperability framework to develop IEEE-based standards on power system applications and control through the use of communication infrastructures. The first of this series IEEE Std 2030 (2011) presents communication and information networks interfaces for different domains of the smart grid. Moreover, this reference model presents the communication requirements for each interface (e.g., security, availability, latency etc.).

In addition, the IEEE P2030.1 Working Group [129] develops a draft guide for electric-sourced transportation infrastructures. Also, P2030 task force-3 defines communication requirements between devices in the smart grid. They are going to describe the network, transport, and session layers (from OSI reference model). Recently, IEEE has established a new technical advisory group (IEEE 802.24) which will work with multiple IEEE 802 working group standards of which are very essential for smart grid communications [130].

\subsection{Measurement-based studies}

Previous paragraphs show that wide-area wireless communication technologies will be predominant role in EV network communications. On the other hand, since the number of mobile internet users has flourished, the user experience deviated significantly from theoretical results. Hence, there is a need for detailed measurement based studies to understand and predict the performance of the wireless technology and quantify the effects of performance degradation.

There are only a handful of measurement-based studies that focuses on the performance of the wireless network (WiFi, 3F (UMTS), EV-DO, and WiMAX) [131-133]. In [133], authors conducted a measurement study to evaluate the performance of the mobile Internet access with 3G (UMTS) and WiFi networks. The measurement was carried out in Seattle, San Francisco, and Amherst. Across all cities, the average availability of $3 \mathrm{G}$ and WiFi is $87 \%$ and $11 \%$, respectively. The details of their findings is presented in Table 7. Then, they proposed a hybrid framework to improve the availability of $3 G$ by augmenting it with WiFi.

Similarly, [132] presents an architecture to improve end user experience by exploiting (i) channel diversity, (ii) wireless network service provider diversity, and (iii) technology diversity (UMTS, CDMA, etc.). Their results shows that the proposed Mobile Access Router architecture decreases the blackout periods considerably and increases average throughput. In addition, [131] shows the results of a city-wide mobile Internet experimentation results. The mobile nodes in their test bed employs both EV-DO and WiFi interfaces. Their focus is on measuring the signal latency and TCP throughput performance. Their results indicate that average latencies varies between 150 to $400 \mathrm{~ms}$ and mobile TCP throughput is around 752 Kbps.

Table 7 Availability performance of wide area wireless technologies [133]

\begin{tabular}{|c|c|c|c|c|c|}
\hline & \multicolumn{2}{|c|}{ Amherst } & \multicolumn{2}{|c|}{ Seattle } & \multirow{2}{*}{$\begin{array}{c}\text { San Francisco } \\
\text { Average }\end{array}$} \\
\hline & Average & Peak & Average & Peak & \\
\hline 3G (UMTS) & $90 \%$ & $85.5 \%$ & $82 \%$ & $79 \%$ & $89 \%$ \\
\hline WiFi & $12 \%$ & $10 \%$ & $10 \%$ & $8.5 \%$ & $11 \%$ \\
\hline
\end{tabular}




\section{Conclusions}

In this paper, we provided a survey of the communication requirements and technologies for the Internet of electric vehicles. First, we presented the current status of the power grid. We specifically focused on the power generation and distribution networks. We identified the challenges introduced by the projected EV demand. Then, we showed that the EV demand may have disruptive effects in the current information and the IoEV infrastructures that are needed to support, control, and manage the energy transfer between vehicles and the power. Next, we grouped related smart grid applications and surveyed the communication requirements, standards, and candidate technologies for each group. We showed that in the absence of two-way communications, the proliferation of EVs will pose threats to the existing power grid and will not reach projected mainstream success.

In the future, we plan to expand our research in the following ways. The choice of communication technology and standards should consider the performance of the each candidate. It is also worth noting that the importance of performance evaluation will increase as the EVs gain widespread acceptance. For instance, if a central authority receives a few queries (location and pricing information for public charging stations) per minute, the cost of communication delays, unavailability, etc. will be negligible. On the other hand, as the query rate increases, underlying infrastructure should provide high availability and low latency. Thus, it is crucial to quantify the effects of the underlying communication technology.

\section{Competing interests}

The authors declare that they have no competing interests.

\section{Author details}

${ }^{1}$ Qatar Environment and Energy Research Institute, Qatar Foundation, PO Box 5825, Doha, Qatar. ${ }^{2}$ Computer and Information Technology, Purdue University, West Lafayette, IN 47907, USA.

Received: 30 August 2014 Accepted: 24 November 2014 Published: 16 December 2014

\section{References}

1. Electrification roadmap: Revolutionizing transportation and achieving energy security. Technical report (2009)

2. JG Kassakian, R Schmalensee, The future of the electric grid: an interdisciplinary MIT study. Technical report, Technical report, Massachusetts Institute of Technology (2011)

3. Technology roadmap: Electric and plug-in hybrid electric vehicles. Technical report, International Energy Agency (June 2011)

4. Tesla To Build National Electric Car Charging Network. http://www forbes.com/sites/toddwoody/2012/09/25/tesla-to-build-nationalelectric-car-charging-network/ [Accessed: Aug 2014]

5. ABB Wins Tender for Europes Largest Electric Vehicle Fast-charging Network. http://www.abb.com/cawp/seitp202/d07e075541462e04c 125798000353578.aspx [Accessed: Aug 2014]

6. Electric vehicles charging equipments. Technical report, Pike Research (2011)

7. Y Yan, Y Qian, H Sharif, D Tipper, A survey on smart grid communication infrastructures: Motivations, requirements and challenges. IEEE Commun. Surv. Tutor. 15(1), 1-16 (2012)
8. V Gungor, D Sahin, T Kocak, S Ergut, C Buccella, C Cecati, G Hancke, A survey on smart grid potential applications and communication requirements. IEEE Trans. Ind. Inform. 9(1) (2012)

9. W Su, H Eichi, W Zeng, MY Chow, A survey on the electrification of transportation in a smart grid environment. IEEE Trans. Ind. Inform. 9(1), $1-10(2012)$

10. W Wang, Y Xu, M Khanna, A survey on the communication architectures in smart grid. Comput. Netw. 55(15), 3604-3629 (2011)

11. IS Bayram, G Michailidis, M Devetsikiotis, B Parkhideh, in Proc. IEEE International Conference on Smart Grid Communications. Strategies for competing energy storage technologies for in dc fast charging stations (Tainan City, Taiwan, 2012), pp. 1-6

12. IS Bayram, G Michailidis, M Devetsikiotis, in Proc. IEEE International Conference on Smart Grid Communications. Electric power resource provisioning for large scale public EV charging facilities (Vancouver, Canada, 2013)

13. E Bloom, Global building stock database: Commectical and residential building floor space by country and building type (2011-2012). Technical report, Pike Research (2012)

14. MC Falvo, D Sbordone, Bayram I S, M Devetsikiotis, in Proc. IEEE International Symposium on Power Electronics, Electrical Drives, Automation and Motion. EV charging stations and modes: international standards (Naples, Italy, 2014)

15. Transitions to Alternative Transportation Technologies-Plug-in Hybrid Electric Vehicles. The National Academies Press (2010). http://www.nap. edu/openbook.php?record_id=12826

16. GT Heydt, R Ayyanar, KW Hedman, V Vittal, Electric power and energy engineering: the first century. Proc. IEEE. 100(Special Centennial Issue), 1315-1328 (2012)

17. GW Arnold, Challenges and opportunities in smart grid: a position article. Proc. IEEE. 99(6), 922-927 (2011)

18. B Wollenberg, A Wood, Power generation, operation and control. (John Wiley\&Sons, Inc, 1996), pp. 264-327

19. M Scott, M Meyer, D Elliot, W Warwick, Impacts of plug-in hybrid vehicles on electric utilities and reginal US power grids. Technical report, Pasific Northwest National Laboratory, Palo Alto, CA (2007)

20. P Denholm, W Short, An evaluation of utility system impacts and benefits of optimally dispatched plug-in hybrid electric vehicles. Technical report, National Renewable Energy Laboratory (2006)

21. K Parks, P Denholm, T Markel, Costs and emissions associated with plug-in hybrid electric vehicle charging in the Excel energy colorado service territory. Technical report, National Renewable Energy Laboratory (2007)

22. SRW Letendre, M Cross, Plug-in hybrid vehicles the vermont grid: a scoping analysis. Technical report, University of Vermont Transportation Center (2008)

23. A Shortt, OM Malley, Quantifying the long-term impact of electric vehicles on the generation portfolio. IEEE Trans. Smart Grid. 5(1), 71-83 (2014)

24. WH Hadley, Impact of plug-in hybrid vehicles on the electric grid. Technical report, Oak Ridge National Labs (October 2006)

25. A Ipakchi, F Albuyeh, Grid of the future. IEEE Power Energy Mag 7(2), 52-62 (2009)

26. W Shireen, S Patel, in Proc. IEEE PES Transmission and Distribution Conference and Exposition. Plug-in hybrid electric vehicles in the smart grid environment (IEEE, 2010), pp. 1-4

27. K Valentine, WG Temple, KM Zhang, Intelligent electric vehicle charging: rethinking the valley-fill. J. Power Sources. 196(24), 10717-10726 (2011)

28. L Gan, U Topcu, S Low, in Proc. IEEE Conference on Decision and Control and European Control Conference. Optimal decentralized protocol for electric vehicle charging, (2011), pp. 5798-5804

29. S Abraham, National transmission grid study. Technical report (2002)

30. C Liu, KT Chau, D Wu, S Gao, Opportunities and challenges of vehicle-to-home, vehicle-to-vehicle, and vehicle-to-grid technologies. Proc. IEEE. 101(11), 2409-2427 (2013)

31. W Tushar, W Saad, HV Poor, DB Smith, Economics of electric vehicle charging: a game theoretic approach. IEEE Trans. Smart Grid. 3(4), 1767-1778 (2012)

32. Y Wang, W Saad, Z Han, HV Poor, T Basar, A game-theoretic approach to energy trading in the smart grid. IEEE Trans. Smart Grid. 5(3), 1439-1450 (2014) 
33. P Yi, T Zhu, B Jiang, B Wang, D Towsley, in Proc. IEEE International Conference on Communications. An energy transmission and distribution network using electric vehicles, (2012), pp. 3335-3339

34. K Clement-Nyns, E Haesen, J Driesen, The impact of charging plug-in hybrid electric vehicles on a residential distribution grid. IEEE Trans. Power Syst. 25(1), 371-380 (2010)

35. S Shao, M Pipattanasomporn, S Rahman, in Proc. IEEE Power Energy Society General Meeting. Challenges of PHEV penetration to the residential distribution network, (2009), pp. 1-8

36. A Kwasinski, A Kwasinski, Signal processing in the electrification of vehicular transportation: techniques for electric and plug-in hybrid electric vehicles on the smart grid. IEEE Signal Process. Mag. 29(5), 14-23 (2012)

37. MD Galus, MG Vayá, T Krause, G Andersson, The role of electric vehicles in smart grids.Wiley Interdiscip. Rev. Energy Environ. 2(4), 384-400 (2013)

38. K Clement-Nyns, E Haesen, J Driesen, The impact of charging plug-in hybrid electric vehicles on a residential distribution grid. IEEE Trans. Power Syst. 25(1), 371-380 (2010)

39. How the smart grid enables utilities to integrate electric vehicles (white paper). Silver Spring Networks (2012)

40. RA Verzijlbergh, MO Grond, Z Lukszo, JG Slootweg, MD llic, Network impacts and cost savings of controlled EV charging. IEEE Trans. Smart Grid. 3(3), 1203-1212 (2012)

41. C Quinn, D Zimmerle, TH Bradley, The effect of communication architecture on the availability, reliability, and economics of plug-in hybrid electric vehicle-to-grid ancillary services. J. Power Sour. 195(5), 1500-1509 (2010)

42. E Sortomme, MM Hindi, SDJ MacPherson, S Venkata, Coordinated charging of plug-in hybrid electric vehicles to minimize distribution system losses. IEEE Trans. Smart Grid. 2(1), 198-205 (2011)

43. L Jian, GXu H Xue, X Zhu, D Zhao, ZY Shao, Regulated charging of plug-in hybrid electric vehicles for minimizing load variance in household smart microgrid. IEEE Trans. Ind. Electron. 60(8), 3218-3226 (2013)

44. N Rotering, M llic, Optimal charge control of plug-in hybrid electric vehicles in deregulated electricity markets. IEEE Trans. Power Syst. 26(3), 1021-1029 (2011)

45. J Wang, C Liu, D Ton, Y Zhou, J Kim, A Vyas, Impact of plug-in hybrid electric vehicles on power systems with demand response and wind power. Energy Policy. 39(7), 4016-4021 (2011)

46. M Yilmaz, PT Krein, Review of the impact of vehicle-to-grid technologies on distribution systems and utility interfaces. IEEE Trans. Power Electron. 28(12), 5673-5689 (2013)

47. XXi, R Sioshansi, Using price-based signals to control plug-in electric vehicle fleet charging. IEEE Trans. Smart Grid. 5, 1-15 (2014)

48. P Samadi, A-H Mohsenian-Rad, R Schober, WWS Wong, J Jatskevich, in Proc. IEEE International Conference on Smart Grid Communications. Optimal real-time pricing algorithm based on utility maximization for smart grid (Washington D.C., USA, 2010), pp. 415-420

49. IS Bayram, M Abdallah, K Qaraqe, in Proc. IEEE International Energy Conference. Providing QoS guarantees to multiple classes of EVs under deterministic grid power, (2014)

50. L Chen, H Suzuki, T Wachi, Y Shimura, Components of nodal prices for electric power systems. IEEE Trans. Power Syst. 17(1), 41-49 (2002)

51. IS Bayram, G Michailidis, M Devetsikiotis, F Granelli, Electric power allocation in a network of fast charging stations. IEEE J. Selected Areas Commun. 31(7), 1235-1246 (2013)

52. M Alizadeh, A Scaglione, RJ Thomas, From packet to power switching: digital direct load scheduling. IEEE J. Selected Areas Commun. 30(6), 1027-1036 (2012)

53. AY Saber, GK Venayagamoorthy, Efficient utilization of renewable energy sources by gridable vehicles in cyber-physical energy systems. IEEE Syst. J. 4(3), 285-294 (2010)

54. H Lu, G Pang, G Kesidis, IE, CS\&E and EE Depts, Automated scheduling of deferrable PEV/PHEV load in the smart grid.Technical report. Technical Report CSE-12-004, Pennsylvania State University, CSE Dept (2012)

55. RA Waraich, 1 plug-in hybrid electric vehicles and smart grid: investigations based on a micro-simulation Transportation Res. Part C: Emerging Technol. 28, 74-86 (2013)

56. K Clement, E Haesen, J Driesen, in Proc. IEEE Power Systems Conference and Exposition. Coordinated charging of multiple plug-in hybrid electric vehicles in residential distribution grids, (2009), pp. 1-7
57. S Chen, $\mathrm{Y} \mathrm{Ji}, \mathrm{L}$ Tong, in Proc. IEEE Power and Energy Society General Meeting. Large scale charging of electric vehicles, (2012), pp. 1-9

58. SChen, Y Ji, L Tong, in Proc. IEEE Sensor Array and Multichannel Signal Processing Workshop. Deadline scheduling for large scale charging of electric vehicles with renewable energy, (2012), pp. 13-16

59. I Bayram, G Michailidis, I Papapanagiotou, M Devetsikiotis, in Proc. IEEE International Global Communication Conference. Decentralized control of electric vehicles in a network of fast charging stations (Atlanta, GA, 2013)

60. IS Bayram, G Michailidis, M Devetsikiotis, Unsplittable load balancing in a network of charging stations under QoS guarantees. IEEE Trans. Smart Grid. 6, 1-11 (2014)

61. L Gan, U Topcu, S Low, in Proc. IEEE Conference on Decision and Control and European Control Conference. Optimal decentralized protocol for electric vehicle charging, (2011), pp. 5798-5804

62. DS Callaway, IA Hiskens, Achieving controllability of electric loads. Proc IEEE. 99(1) (2011)

63. Z Ma, D Callaway, I Hiskens, in Decision and Control (CDC), 2010 49th IEEE Conference On. Decentralized charging control for large populations of plug-in electric vehicles (IEEE, 2010), pp. 206-212

64. N Rotering, M llic, Optimal charge control of plug-in hybrid electric vehicles in deregulated electricity markets. IEEE Trans. Power Syst. 26(3), 1021-1029 (2011)

65. MD Galus, G Andersson, in Proc. IEEE Energy 2030 Conference. Demand management of grid connected plug-in hybrid electric vehicles (phev), (2008), pp. 1-8

66. IS Bayram, M Ismail, M Abdallah, K Qaraqe, E Serpedin, in IEEE International Conference on Smart Grid Communications. A pricing-based load shifting framework for EV fast charging stations, (2014)

67. L Gan, U Topcu, SH Low, in Proc. IEEE Power and Energy Society General Meeting. Stochastic distributed protocol for electric vehicle charging with discrete charging rate, (2012), pp. 1-8

68. L Gan, U Topcu, S Low, in Proc. IEEE Decision and Control and European Control Conference. Optimal decentralized protocol for electric vehicle charging, (2011), pp. 5798-5804

69. Z Ma, DS Callaway, IA Hiskens, Decentralized charging control of large populations of plug-in electric vehicles. IEEE Trans. Control Syst. Technol. 21(1), 67-78 (2013)

70. Z Fan, in Proc. IEEE International Conference on Smart Grid Communications. Distributed charging of PHEV in a smart grid, (2011), pp. 255-260

71. W Su, M-Y Chow, in Proc. IEEE Power and Energy Society General Meeting. Performance evaluation of a PHEV parking station using particle swarm optimization, (2011), pp. 1-6

72. A Deshpande, P Murali, in Control Conference (ECC), 2013 European. Pricing long-term permits and scheduling of electric vehicle charging in parking lots with shared resources (IEEE, 2013), pp. 3584-3589

73. L Jian, H Xue, G Xu, X Zhu, D Zhao, ZY Shao, Regulated charging of plugin hybrid electric vehicles for minimizing load variance in household smart microgrid. IEEE Trans. Ind. Electron. 60(8), 3218-3226 (2013)

74. SAE Vehicle electrictification standards. http://www.sae.org/smartgrid/ [Accessed: June 2014]

75. K Gowri, RG Pratt, FK Tuffner, MCW Kintner-Meyer, Vehicle to grid communication standards development, testing and validation: status report. Pacific Northwest National Laboratory, Technical report (2011)

76. T Bohn, H Chaudhry, in Proc. IEEE PES Innovative Smart Grid Technologies. Overview of SAE standards for plug-in electric vehicle, (2012), pp. 1-7

77. S Aman, Y Simmhan, VK Prasanna, Energy management systems: state of the art and emerging trends. IEEE Commun. Mag. 51(1), 114-119 (2013)

78. L Bartram, J Rodgers, K Muise, Chasing the negawatt: visualization for sustainable living. Proc. IEEE Comput. Graph. Appl. Conf. 30(3), 8-14 (2010)

79. Zigbee Alliance. http://www.zigbee.org/ [Accessed: Aug. 2014]

80. J Mu, A minimum physical distance delivery protocol based on zigbee in smart grid. EURASIP J. Wireless Commun. Netw. 2014(1), 108 (2014)

81. E Bou-Harb, C Fachkha, M Pourzandi, M Debbabi, C Assi, Communication security for smart grid distribution networks. IEEE Commun. Mag. 51(1), 42-49 (2013)

82. Standardization roadmap for electric vehicles. Technical report, American National Standards Institute (April 2012)

83. S Galli, A Scaglione, Z Wang, in Proc. IEEE International Conference on Smart Grid Communications. Power line communications and the smart grid, (2010), pp. 303-308 
84. S Galli, A Scaglione, Z Wang, For the grid and through the grid: the role of power line communications in the smart grid. Proc. IEEE. 99(6), 998-1027 (2011)

85. P Kulkarni, S Gormus, Z Fan, B Motz, A mesh-radio-based solution for smart metering networks. IEEE Commun. Mag. 50(7), 86-95 (2012)

86. O Fatemieh, R Chandra, CA Gunter, in Proc. IEEE International Symposium on Resilient Control Systems. Low cost and secure smart meter communications using the tv white spaces, (2010), pp. 37-42

87. D Lekomtcev, R Maršálek, Comparison of 802.11 af and 802.22 standards - physical layer and cognitive functionality. Elektro Revue. 3(2), 12-18 (2012)

88. AB Flores, RE Guerra, EW Knightly, P Ecclesine, S Pandey, leee 802.11af: a standard for TV white space spectrum sharing. IEEE Commun. Mag. 51(10), 92-100 (2013)

89. Smart Grid DSL and QWEST Team Up. http://gigaom.com/cleantech/ smart-grid-dsl-current-and-qwest-team-up/ [Accessed: Aug. 2014]

90. Plugshare- EV Charging Station Map. www.plugshare.com [Accessed Jan. 2014]

91. Electric Vehicle Charging. www.chargepoint.com [Accessed: Oct. 2013]

92. J Pkrzywa, SAE ground vehicle standards smart grid. SAE Taipei. Available: http://sae-taipei.org.tw/image/1283265726.pdf

93. B Bangerter, S Talwar, R Arefi, K Stewart, Networks and devices for the $5 \mathrm{G}$ era. IEEE Commun. Mag. 52(2), 90-96 (2014)

94. IEEE draft guide for smart grid interoperability of energy technology and information technology operation with the electric power system (EPS), and end-use applications and loads. IEEE P2030/D5.0 February 2011 1-126 (2011)

95. ML Sichitiu, in Proceedings of World Wireless Congress. Wireless mesh networks: opportunities and challenges, (2005)

96. Tropos Networks. gridcom.tropos.com [Accessed: Aug. 2014]

97. Volkswagen Research. www.wireless-wolfsburg.de [Accessed: July 2014]

98. M Burns, Interoperability knowledge base. http://collaborate.nist.gov/ twiki-sggrid/bin/view/SmartGrid/InteroperabilityKnowledgeBase

99. Edison Smartconnect - Industry resource center: 2008-2009 smart grid use cases. http://www.sce.com/CustomerService/smartconnect/ industry-resource-center/smartgrid-usecase.htm

100. E Hossain, Z Han, HV Poor, Smart Grid Communications and Networking. (Cambridge Univ. Press, Cambridge, UK, 2012)

101. IS Bayram, G Michailidis, M Devetsikiotis, S Bhattacharya, A Chakrabortty, F Granelli, in Proc. IEEE International Conference on Smart Grid Communications. Local energy storage sizing in plug-in hybrid electric vehicle charging stations under blocking probability constraints, (2011) pp. $78-83$

102. IS Bayram, G Michailidis, M Devetsikiotis, F Granelli, S Bhattacharya, in Control and Optimization Methods for Electric Smart Grids. Power Electronics and Power Systems, vol. 3, ed. by A Chakrabortty, MD Ilic. Smart vehicles in the smart grid: challenges, trends, and application to the design of charging stations (Springer, 2012), pp. 133-145

103. D Niyato, P Wang, E Hossain, Reliability analysis and redundancy design of smart grid wireless communications system for demand side management. IEEE Wireless Commun. 19(3), 38-46 (2012)

104. S Dharmaraja, $V$ Jindal, U Varshney, Reliability and survivability analysis for UMTS networks: an analytical approach. IEEE Trans. Netw. Serv. Manag. 5(3), 132-142 (2008)

105. AP Snow, U Varshney, AD Malloy, Reliability and survivability of wireless and mobile networks. Computer. 33(7), 49-55 (2000)

106. A Bruce, in Proc. IEEE Power Industry Computer Applications. Reliability analysis of electric utility SCADA systems, (1997), pp. 200-205

107. S-I Inage, Modeling load shifting using electric vehicles in a smart grid environment. Technical report, OECD Publishing (2010)

108. H Li, W Zhang, in Proc. IEEE Global Telecommunications Conference. QoS routing in smart grid, (2010), pp. 1-6

109. Mobile broadband capacity constraints the need for optimization. Technical report, RYSAVY Research (2010)

110. I Papapanagiotou, D Toumpakaris, J Lee, M Devetsikiotis, A survey on next generation mobile WiMAX networks: objectives, features and technical challenges. IEEE Commun. Surv. Tutor. 11(4), 3-18 (2009)

111. GS Paschos, I Papapanagiotou, CG Argyropoulos, SA Kotsopoulos, in 45th Congress FITCE. A heuristic strategy for ieee 802.16 WiMAX scheduler for quality of service, (2006)
112. C-X Wang, F Haider, X Gao, X-H You, Y Yang, D Yuan, H Aggoune, H Haas, S Fletcher, E Hepsaydir, Cellular architecture and key technologies for $5 \mathrm{G}$ wireless communication networks. IEEE Commun. Mag. 52(2), 122-130 (2014)

113. I Papapanagiotou, GS Paschos, SA Kotsopoulos, M Devetsikiotis. Proc. IEEE Global Telecommunications Conference, (2007), pp. 2530-2535

114. I Papapanagiotou, GS Paschos, M Devetsikiotis, A comparison performance analysis of QoS WLANS: approaches with enhanced features. Adv. Multimedia. 2007(1), 1 (2007)

115. GS Paschos, I Papapanagiotou, SA Kotsopoulos, GK Karagiannidis, A new MAC protocol with pseudo-TDMA behavior for supporting quality of service in 802.11 wireless LANs. EURASIP J. Wirel. Commun. Netw. 2006(3), 8-189 (2006)

116. I Papapanagiotou, JS Vardakas, GS Paschos, MD Logothetis, SA Kotsopoulos, in Proceedings of the 3rd International Conference on Mobile Multimedia Communications. MobiMedia '07. Performance evaluation of IEEE 802.11e based on ON-OFF traffic model (ICST Brussels, Belgium, Belgium, 2007), pp. 17-1176. http://dl.acm.org/citation.cfm?id=1385289. 1385310

117. Y Yan, Y Qian, H Sharif, D Tipper, A survey on cyber security for smart grid communications. IEEE Commun. Surv. Tutor. 14(4), 998-1010 (2012)

118. J Liu, Y Xiao, S Li, W Liang, CLP Chen, Cyber security and privacy issues in smart grids. IEEE Commun. Surv. Tutor. 14(4), 981-997 (2012)

119. H Su, M Qiu, H Wang, Secure wireless communication system for smart grid with rechargeable electric vehicles. IEEE Commun. Mag. 50(8), 62-68 (2012)

120. Y Mo, TH-J Kim, K Brancik, D Dickinson, H Lee, A Perrig, B Sinopoli, Cyber 2013; physical security of a smart grid infrastructure. Proc. IEEE. 100(1), 195-209 (2012)

121. Roadmap to achieve energy delivery systems cyber security. Technical report, The US Department of Energy (2011)

122. NISTIR 7628, Guidelines for smart grid cyber security strategy, Architecture, and High-Level Requirements. Technical report, National Institute of Standards and Technology

123. M Qiu, H Su, M Chen, Z Ming, LT Yang, Balance of security strength and energy for a PMU monitoring system in smart grid. IEEE Commun. Mag. 50(5), 142-149 (2012)

124. AC-F Chan, Z Zhou, On smart grid cybersecurity standardization: issues of designing with NISTIR 7628. IEEE Commun. Mag. 51(1), 58-65 (2013)

125. P-Y Chen, S-M Cheng, K-C Chen, Smart attacks in smart grid communication networks. IEEE Commun. Mag. 50(8), 24-29 (2012)

126. E-K Lee, M Gerla, SY Oh, Physical layer security in wireless smart grid. IEEE Commun. Mag. 50(8), 46-52 (2012)

127. B Karimi, V Namboodiri, in Proc. IEEE Conference on Computer Communications Workshops. Capacity analysis of a wireless backhaul for metering in the smart grid, (2012), pp. 61-66

128. N Framework, Roadmap for smart grid interoperability standards. National Institute of Standards and Technology (2010)

129. IEEE P2030.1 Draft Guide for Electric-Sourced Transportation Infrastructure. http://grouper.ieee.org/groups/scc21/2030.1/2030. 1_index.html [Accessed: Aug. 2014]

130. IEEE 802.24 Smart Grid Technical Advisory Group. http://standards.iee. org/news/2012/802.24tag.html

131. J Ormont, J Walker, S Banerjee, A Sridharan, M Seshadri, S Machiraju, in Proceedings of the Third ACM International Workshop on Wireless Network Testbeds, Experimental Evaluation and Characterization. A city-wide vehicular infrastructure for wide-area wireless experimentation (ACM, 2008), pp. 3-10

132. J Ott, D Kutscher, in Proc. IEEE Computer and Communications Societies. Proceedings, vol. 3. A disconnection-tolerant transport for drive-thru internet environments, (2005), pp. 1849-1862

133. A Balasubramanian, R Mahajan, A Venkataramani, in Proceedings of the 8th International Conference on Mobile Systems, Applications, and Services. Augmenting mobile 3G using WiFi, (2010), pp. 209-222

doi:10.1186/1687-1499-2014-223

Cite this article as: Bayram and Papapanagiotou: A survey on communication technologies and requirements for internet of electric vehicles. EURASIP Journal on Wireless Communications and Networking 2014 2014:223 